\title{
Comparison of Gray Scale and Color Doppler Sonography with Cytopathology Findings in Cervical Lymphadenopathy in Tertiary Level Hospital
}

\section{Regmi D $D^{1}$, Lohani $B^{2}$, Kayastha $P^{2}$, Shreevastav $S^{3}$, Paudel $S^{2}$, Jha $S^{1}$, Bhatta $\mathbf{U}^{4}$}

\author{
${ }^{1}$ Department of Radiology and Imaging, Kanti Children's Hospital, Kathmandu, Nepal \\ ${ }^{2}$ Department of Radiology and Imaging, Tribhuwan University Teaching Hospital, Kathmandu, Nepal \\ ${ }^{3}$ Department of Pathology, Tribhuwan University Teaching Hospital, Kathmandu, Nepal \\ ${ }^{4}$ Department of Pathology, Kanti Children's Hospital, Kathmandu, Nepal
}

Received: June 15, 2019

Accepted: September 30, 2019

Published: December 31, 2019

Cite this paper:

Regmi D, Lohani B, Kayastha P et al. Comparison Of Gray Scale And Color Doppler Sonography with Cytopathology Findings in Cervical Lymphadenopathy in Tertiary Level Hospital. Nepalese Journal of Radiology 2019;9(14):10-16.http://dx.doi.org/10.3126/njr.v9i2.27414

\begin{abstract}
Introduction: Cervical region is the commonest area of lymphadenopathy which is easily accessible to ultrasound and Doppler study. The morphological and vascular-architectural differences among various nodal diseases aids in differentiating benign from malignant causes.
\end{abstract}

Methods: The study was done on the 108 patients referred to Department of Radiology and Imaging, TUTH for ultrasound of cervical lymphadenopathy who subsequently underwent FNAC examination. Gray scale evaluation for morphology of the nodes along with Doppler evaluation for resistive index (RI), pulsatility index (PI) and Peak systolic velocity (PSV) were done and correlated with FNAC findings.

Results: Among the 108 lymph nodes, 24 were proven to be malignant on FNAC. Features such as $\mathrm{S} / \mathrm{L}$ ratio $>0.5$, absence of echogenic hilum, and abnormal vascular pattern demonstrated sensitivities of $96 \%, 92 \%$, and $87 \%$, specificities of $74 \%, 65 \%$ and $77 \%$ and positive predictive values (PPVs) of 51\%, 43\%, and 55\% respectively. The cutoff values for RI, PI and PSV were found to be $0.705,1.34$ and $17.5 \mathrm{~cm} / \mathrm{s}$ with sensitivities of $96 \%, 96 \%$ and $87 \%$, specificities of $95 \%, 99 \%$ and $88 \%$ and positive predictive values (PPVs) of $85 \%, 95 \%$ and $70 \%$ respectively.

Conclusion: Ultrasound findings of S/L ratio, absence of echogenic hilum, abnormal vascular pattern and Doppler indices revealed good sensitivity, specificity, and accuracy in differentiating benign and malignant lymph nodes.

Keywords: Lymphadenopathy; Lymph Nodes; Ultrasonography

Correspondence to: Dr. Dosti Regmi

Department of Radiology and Imaging

Kanti Children's Hospital

Kathmandu, Nepal

Email: dostiregmi27@gmail.com

\section{INTRODUCTION}

Cervical region is the commonest area 
of lymphadenopathy in several reactive, tubercular, malignant and metastatic diseases. Ultrasound with color Doppler is a safe and widely available tool for detection and characterization of cervical lymph nodes. Cervical lymph nodes with the advantage of being in superficial location have better spatial resolution on ultrasound and are superior to CT and MRI. ${ }^{1}$

There will be morphological and vasculararchitectural differences among various nodal diseases which aid in differentiating benign from malignant causes. The histological changes seen within the node have been used to explain morphologic changes on gray-scale sonography. The normal vascular morphology in metastatic nodes is destroyed by neoplastic infiltration, whereas in inflammatory disease there will be dilatation of the intranodal vessels. These changes can be depicted with the use of color or power Doppler indices like RI, PI and PSV. ${ }^{2}$

Accurate differentiation between benign and malignant lymph nodes can decrease the number of patient undergoing unnecessary invasive procedures like FNAC and biopsy.

\section{METHODS}

A prospective quantitative study was done on 108 patients > 16 yrs who were referred to Department of Radiology and Imaging, TUTH for ultrasound of cervical lymphadenopathy and subsequently underwent FNAC of the nodes. The study was done from July 2017 to July 2018. Ethical approval was obtained from the Institutional Review Board. All the participants selected as per inclusion criteria were explained about the study. An informed written consent was taken from each patient. For ultrasound examination the patients were asked to lie supine extending their neck. A 7.5$10 \mathrm{MHz}$ linear probe was used for evaluating the lymph nodes. Sonographic evaluation was performed in the largest node followed by FNAC of the same node.

Morphological assessment included gray scale evaluation of short axis to long axis $(\mathrm{S} / \mathrm{L})$ ratio and the presence or absence of the echogenic hilum. Doppler examination was performed with sample gate kept in the center of the vessel, and the angle of insonation was kept at $<60^{\circ}$. Vascularity pattern (central, peripheral, mixed or avascular), Resistivity Index (RI), Pulsatility Index (PI) and Peak systolic Velocity (PSV) was studied. ${ }^{2,3,4}$ Following FNAC, cytopathology report was obtained from Department of Pathology.

The sensitivity, specificity, PPV, NPV and accuracy of different morphological features and Doppler indices in differentiating malignant from benign nodes were obtained with contingency tables. The significance of differences in S/L ratio, vascularity pattern and Doppler indices in benign and malignant lymph nodes along with the cut off values of Doppler indices were assessed with ROC (Receiver operating characteristic) curve.

\section{RESULTS}

We evaluated a total of 108 patients ( 52 male, 56 female) who met the selection criteria. Among them 52 were male and 56 were female. The age of the patients ranged from 17 to 84 years. Maximum patients were in the age group of 20-29 years followed by age groups of 16-19 years and 30-39 years. Among the total 108 lymph nodes, 84 were benign (53 reactive and 31 tubercular) and 24 were malignant (18 metastatic and 6 lymphomatous). The most common cause of cervical lymphadenopathy was reactive in 53 patients $(49.1 \%)$, followed by tuberculosis in 31 patients $(28.7 \%)$, metastasis in 18 patients $(16.7 \%)$ and lymphoma in 6 patients $(5.6 \%)$.

\section{ULTRASONOGRAPHIC MORPHOLOGY}

a) Short axis to long axis ratio (S/L ratio):

On gray scale sonography, the $\mathrm{S} / \mathrm{L}$ ratio of benign lymph nodes ranged from 0.33 to 0.9 (Mean 0.56; SD 0.09) and that of malignant lymph nodes ranged from 0.54 to 0.88 (Mean 0.7; SD 0.10).

The S/L ratio $\geq 0.5$ had a sensitivity of $95.83 \%$ and a specificity of $73.80 \%$ with a PPV of $51.11 \%$, a NPV of $94.41 \%$ and an accuracy of $78.70 \%(\mathrm{p}<0.001)$. 
Table 5: Distribution of lymph nodes according to S/L ratio

\begin{tabular}{|c|c|c|c|c|c|}
\hline \multicolumn{1}{|c|}{$\mathrm{S} / \mathrm{L}$} & Reactive & Tuberculosis & Metastasis & Lymphoma & Total \\
\hline$<0.5$ & 43 & 19 & 1 & 0 & 63 \\
\hline$\geq 0.5$ & 10 & 12 & 17 & 6 & 45 \\
\hline Total & 53 & 31 & 18 & 6 & 108 \\
\hline
\end{tabular}

\section{c) Echogenic hilum}

The normal echogenic hilum was absent in $29(34.5 \%)$ of benign and $22(91.7 \%)$ of the malignant lymph nodes. Among benign nodes echogenic hilum was not seen in $25(80 \%)$ of the tubercular nodes but only $4(7 \%)$ of the reactive lymph nodes. Among malignant nodes echogenic hilum was absent in 16 $(88 \%)$ of the malignant nodes and all 6 nodes of lymphoma. Absence of echogenic hilum had sensitivity of $91.7 \%$ and specificity of $65.5 \%$ for the diagnosis of malignancy. It had PPV of $43.1 \%$, NPV of $96.4 \%$ and accuracy of $71.3 \% .(p<0.001)$

\section{DOPPLER ASSESSMENT:}

\section{a) Vascularity pattern}

Seventy five among the 84 benign lymph nodes and all 24 malignant lymph nodes showed some vascularity. Among the benign lymph nodes $9(10.7 \%)$ nodes were avascular (3 reactive and 6 tubercular lymph nodes, 58 (69\%) had central vascularity (50 reactive and 8 tubercular nodes), 12 (14\%) had mixed pattern of vascularity (1 reactive and 11 tubercular) while 5 lymph nodes had peripheral vascularity (all tubercular) (Figure 1).

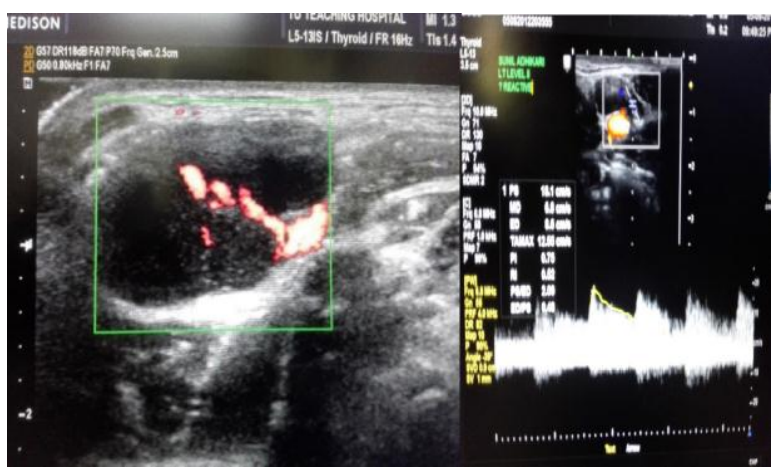

Figure 1: Benign oval reactive lymphnode with central vascularity and low RI.
All the 24 malignant lymph nodes showed some vascularity. Among them 21 (87.5\%) demonstrated either mixed (12 nodes) or peripheral (9 nodes) vascularity whereas remaining $3(12.5 \%)$ nodes demonstrated central vascularity (2 lymphomatous and 1 metastatic node) (Figure 2).

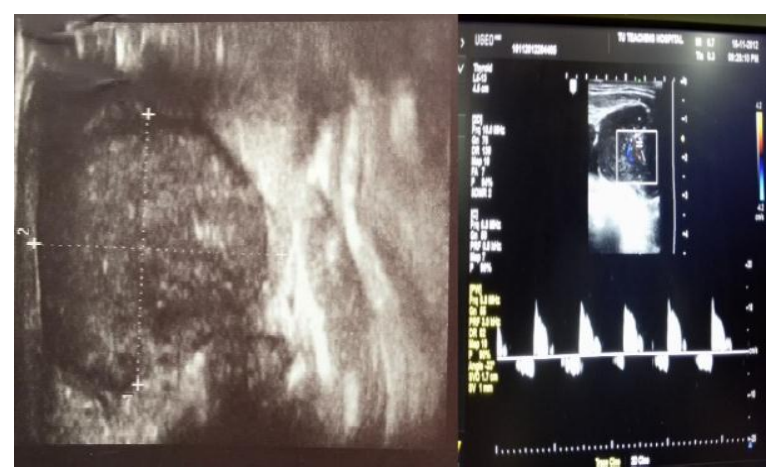

Figure 2: Malignant rounded lymphnode with high RI.

For the nodal vascularity contingency Table 1. was made for benign and malignant lymph nodes vs. central and abnormal vascularity (peripheral or mixed) (Figures 1\&2). Abnormal vascularity recorded a sensitivity of $87.5 \%$ and a specificity of $77.33 \%$ with a PPV of $55.26 \%$, a NPV of $95.08 \%$ and an accuracy of $79.79 \%(p<0.001)$ for the diagnosis of malignancy (Table 1). 


\section{b) Doppler indices}

The mean values of RI, PI and PSV for different types of lymph nodes are shown in Table 2.

The sensitivity and specificity combination points for various indices were derived from the coordinates of the ROC curve to determine the cut-off values. RI above the cutoff value of 0.705 had $96 \%$ sensitivity, $95 \%$ specificity, 85\% PPV, 98\% NPV and 95\% accuracy for diagnosis of malignant lymph node. PI above the cutoff value of 1.34 had $96 \%$ sensitivity, 99\% specificity, 95\% PPV, 98\% NPV and 98\% accuracy for diagnosis of malignant lymph node. Similarly PSV above the cutoff value of $17.5 \mathrm{~cm} / \mathrm{s}$ had $87 \%$ sensitivity, $88 \%$ specificity, $70 \%$ PPV, 96\% NPV and 88\% accuracy for diagnosis of malignant lymph node. These values were statistically significant with $p$ value $<0.001$. The distribution is depicted in Figure 3.

\section{Table 1: Distribution of lvmph nodes according to the pattern of vascularity}

\begin{tabular}{|c|c|c|c|c|c|}
\hline \multirow{2}{*}{ Vascularity } & \multicolumn{2}{|c|}{ Benign } & \multicolumn{2}{c|}{ Malignant } & \multirow{2}{*}{ Total } \\
\cline { 2 - 5 } & Reactive & Tuberculosis & Metastasis & Lymphoma & \\
\hline Absent & 3 & 6 & 0 & 0 & 9 \\
\hline Pentral & 50 & 8 & 1 & 2 & 61 \\
\hline Mixed & 0 & 5 & 9 & 0 & 14 \\
\hline Total & 54 & 11 & 8 & 4 & 24 \\
\hline
\end{tabular}

\section{Table 2: Mean Doppler indices in different types of lymph nodes}

\begin{tabular}{|c|c|c|c|c|c|}
\hline \multirow{2}{*}{$\begin{array}{c}\text { Doppler Indices } \\
\text { Reactive }\end{array}$} & \multicolumn{2}{c|}{ Benign } & \multicolumn{2}{c|}{ Malignant } \\
\cline { 3 - 6 } & Tuberculosis & Metastasis & Lymphoma & \\
\hline RI & Mean & $0.56 \pm 0.09$ & $0.58 \pm 0.09$ & $0.85 \pm 0.08$ & $0.84 \pm 0.05$ \\
\hline PI & Mean & $0.88 \pm 0.19$ & $0.90 \pm 0.14$ & $1.89 \pm 0.35$ & $1.57 \pm 0.12$ \\
\hline PSV & Mean & $13.73 \pm 3.68$ & $11.86 \pm 2.89$ & $21.59 \pm 4.08$ & $21.8 \pm 4.75$ \\
\hline
\end{tabular}
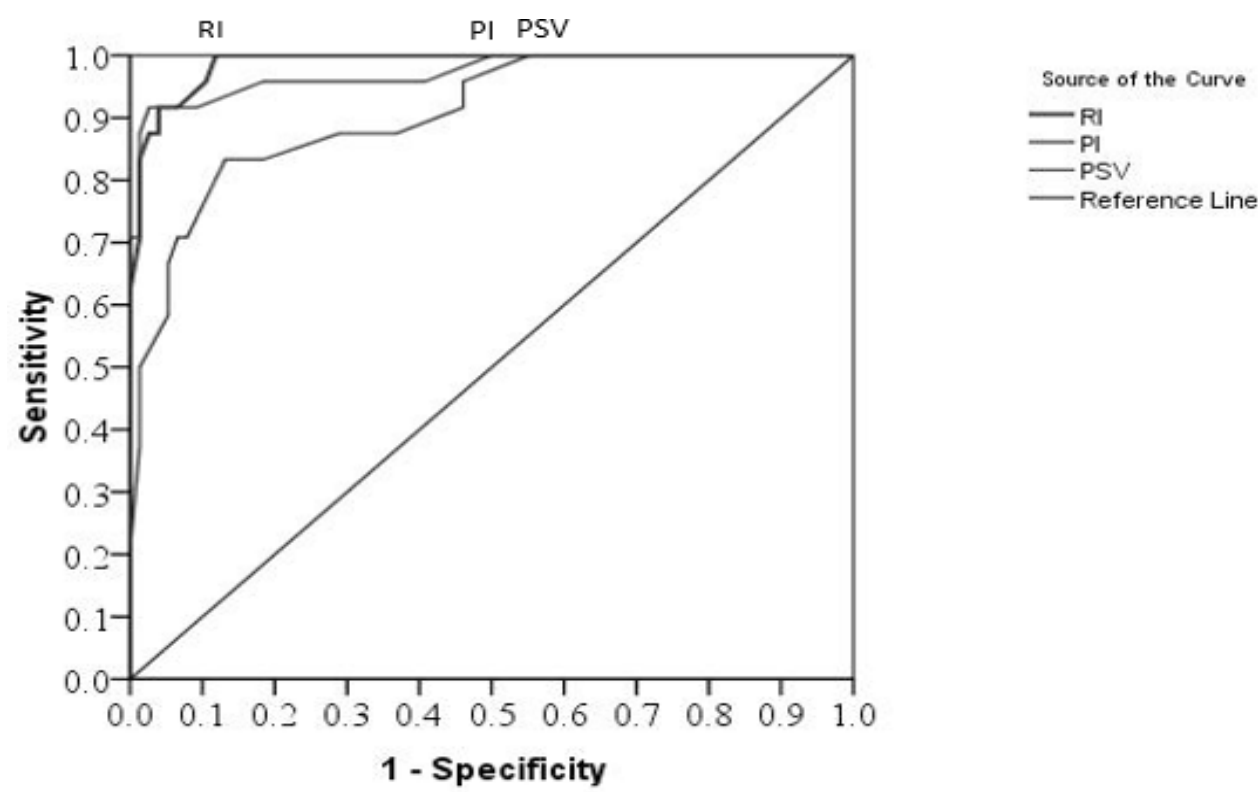

Figure 3: Receiver operating characteristic curve of Doppler indices showing relation between sensitivity and specificity of RI, PI and PSV for lymph nodes. 


\section{DISCUSSION}

The shape of the lymph node was assessed by the S/Lratio (Solbiati index). Elliptical lymph nodes have an $\mathrm{S} / \mathrm{L}$ ratio of $<0.5$ whereas round nodes have $\mathrm{S} / \mathrm{L}$ ratio $>0.5$. In our study round shape showed a higher sensitivity of $95.83 \%$ and relatively lower specificity of $73.80 \%$ for diagnosis of malignancy. Similar findings were observed by Steinkamp et al. ${ }^{5}$ who studied lymph nodes in CT scan of head and neck tumors followed by histologic examination. $\mathrm{S} / \mathrm{L}$ ratio $>0.5$ had $97 \%$ sensitivity; $97 \%$ specificity and $97 \%$ accuracy for malignancy. In another study done by Dong Na DG et al. ${ }^{2}$ in 117 nodes (total 105 patients) with cervical lymphadenopathy an $\mathrm{S} / \mathrm{L}$ ratio $>0.5$ had a sensitivity of $85 \%$ and specificity of $61 \%$ for diagnosis of malignancy.

Metastatic, lymphomatous (Hodgkin's or NonHodgkin's) and tubercular nodes commonly appear round, whereas normal or reactive nodes are usually oval or flat. Metastatic disease can change the shape of the node by infiltrating nodal tissue and expanding the nodal capsule. Thus, round rather than oval nodes are suspicious for malignancy.

\section{Echogenic hilum}

Presence of the echogenic hilum is long considered a sign of benignity. ${ }^{6,7,8}$ Over $90 \%$ of benign cervical nodes with a diameter more than $5 \mathrm{~mm}$ showed echogenic hilum in a study done by Ahuja et al. ${ }^{1}$ In our study, among the 4 reactive lymph nodes without obvious echogenic hilum 3 were small in size $(<0.7 \mathrm{~cm}$ in short axis) and one was suppurative lymphnode. Limited branching and separation of walls of the lymphatic sinuses and blood vessels in smaller nodes could be the reason for inadequate interfaces for reflection of the ultrasound waves to make the hilum echogenic. ${ }^{1}$ Loss of echogenic hilum in the tuberculosis was due to necrosis and these nodes were heteroechoic with the echogenic foci formed by the areas of caseation. ${ }^{1}$ Suppurative lymph nodes also have loss of echogenic hilum.
In the malignant nodes the absence of the echogenic hilum is due to replacement by the tumor cells. Two metastatic lymph nodes in our study showed echogenic hilum. It is possible to see echogenic hilum in early nodal malignancy, because the medullary lymphatic sinuses have not been sufficiently invaded. ${ }^{3}$ In our study absence of echogenic hilum showed higher sensitivity of $91.7 \%$ but relatively lower specificity of $65.5 \%$ for the diagnosis of malignancy which is similar to the findings of Na DG et al. ${ }^{2}$ where they had the sensitivity of $94 \%$ and specificity of only $37 \%$ for malignant nodes.

\section{Vascular Pattern}

In our study most of the malignant nodes demonstrated either peripheral or mixed vascularity which is abnormal vascularity compared to the normal central vascularity except for 2 lymphomatous and 1 metastatic node. The reason could be due to early stage of nodal metastasis which spares the central vessels. In our study, 66\% lymphomatous lymph nodes had preserved central vascularity along with peripheral vascularity. The reason could be because the vessels in the hilum are encased but not narrowed in the lymphoma. Though reactive lymph nodes had characteristic central vascularity, tubercular lymph nodes had variable pattern of vascularity simulating both benign and malignant conditions. Hilar vascularity might be destroyed by the intranodal necrosis, which induces blood supply from the periphery, particularly from inflamed perinodal tissue ${ }^{3,7}$ In the present study, the abnormal vascularity showed a higher sensitivity of $87.5 \%$ and a relatively lower specificity of $77.33 \%$ for the diagnosis of malignancy. Similar study by $\mathrm{Na}$ DG et al. ${ }^{2}$ showed sensitivity of $98 \%$ and specificity of $63 \%$ for the determination of malignancy based on the presence of abnormal nodal vascularity. The sensitivity is higher but the specificity is lower than our study. On the basis of color flow pattern, Dangore SB et $\mathrm{al}^{7}$ showed the sensitivity and specificity to differentiate between benign and malignant 
lymphadenopathy as $87.60 \%$ and $91.66 \%$ respectively. The sensitivity is comparable to our study but had higher specificity than ours. In a study done by Van den Brekel MW etal. ${ }^{8}$ in patients with head and neck squamous cell carcinoma, vascular pattern was valuable in differentiating metastatic from reactive nodes with sensitivity and specificity of $85 \%$ and 93\% respectively. The Doppler sonography study of vascular pattern showed high levels of sensitivity (83\%) and specificity (98\%) in depicting metastatic nodes in another study by Ariji Y etal. ${ }^{9}$

\section{Doppler indices}

In our study the mean RI, PI and PSV were significantly higher in malignant nodes compared to benign lymph nodes. In the study by Dangore et al. ${ }^{7}$ the RI of malignant lymph nodes had a mean value of $0.73 \pm 0.16$ and that of benign nodes had a mean value of 0.65 \pm 0.18 . The PI of malignant lymph nodes had a mean value of $1.76 \pm 0.66$ and that of benign nodes had a mean value of $1.13 \pm 0.48$. The findings in this study were similar to the present study. Similarly the study by Van den Brekel MW et al. ${ }^{8}$ also showed higher mean value of PSV, RI and PI in malignant nodes compared to the benign nodes. In their study mean PSV, RI and PI of metastatic nodes were $21.23 \mathrm{~cm} / \mathrm{s}, 0.79$ and 1.83 respectively while these values were $14.44 \mathrm{~cm} / \mathrm{s}, 0.64$ and 1.18 respectively in reactive nodes. The authors suggested that cell production in metastatic nodes could increase the pressure on internal structures such as vessels and consequently increase the RI and PI. In another study done by Gupta et al. ${ }^{4}$ The RI values in benign lymph nodes ranged from 0.40 to 0.82 with a mean of $0.60 \pm 0.10$. RI values in malignant lymph nodes ranged from 0.56 to 0.88 with a mean of $0.74 \pm 0.08$ which are comparable to our study.

In our study the cutoff value of 0.705 for RI, 1.34 for PI and $17.5 \mathrm{~cm} / \mathrm{s}$ for PSV was determined for diagnosis of malignant lymph node with high sensitivity and specificity. Steinkamp et al. ${ }^{10}$ had suggested optimal cut- off of 0.8 for RI and 1.6 for PI in distinguishing reactive and metastatic nodes had a sensitivity of $80 \%$ and $94 \%$ respectively, and a specificity of $94 \%$ and $97 \%$, respectively. These cut off values were higher than that of the present study but with similar sensitivities and specificities. Ahuja A et al. ${ }^{1}$ found that the optimal cut-off for RI and PI in differentiating reactive from metastatic nodes were 0.7 and 1.4 with a sensitivity of $86 \%$ and $80 \%$, respectively, and a specificity of $70 \%$ and $86 \%$, respectively. ${ }^{11}$ Though the cut off values were similar to our study, the sensitivities and specificities were less. The study by Van den Brekel MW et al. ${ }^{8}$ had cutoff points for RI, PI and PSV as $0.695,1.35$ and $16.5 \mathrm{~cm} / \mathrm{s}$ respectively. It showed sensitivities and specificities of $83 \%$ and $81 \%, 83 \%$ and $68 \%, 72 \%$ and $81 \%$ for RI, PI and PSV respectively. In a study done by $\mathrm{Na}$ DG et al. ${ }^{2}$ the cutoff value of 0.8 for the RI and 1.5 for the PI were $100 \%$ specific for malignancy. However, sensitivities for these cutoff values were only $47 \%$ and $55 \%$ respectively. This high specificity and low sensitivity was suggestive of overlap of the indices in the lower ranges.

\section{CONCLUSION}

The gray scale and color Doppler ultrasound findings of $\mathrm{S} / \mathrm{L}$ ratio, absence of echogenic hilum, abnormal vascular pattern, and Doppler indices revealed good sensitivity, specificity, and accuracy in differentiating benign from malignant lymph nodes.

Morphological features of the nodes such as $\mathrm{S} / \mathrm{L}$ ratio $>0.5$, absence of echogenic hilum, and abnormal vascular pattern and Doppler indices cutoff values 0.705, 1.34 and $17.5 \mathrm{~cm} / \mathrm{s}$ respectively for RI, PI and PSV were sensitive and specific for diagnosis of malignant lymph nodes.

\section{CONFLICT OF INTEREST}

None

\section{SOURCES OF FUNDING}


None

\section{REFERENCES}

1. Ahuja A, Ying M. Sonography of neck lymph nodes. Part II: abnormal lymph nodes. Clin Radiol 2003;58(5):359-366. https://doi. org/10.1016/S0009-9260(02)00584-6

2. Na DG, Lim HK, Byun HS, Kim HD, Ko YH, Baek JH. Differential diagnosis of cervical lymphadenopathy: usefulness of color Doppler sonography. AJR Am J Roentgenol 1997;168(5):1311$1316 . \quad$ https://doi.org/10.2214/ ajr.168.5.9129432

3. Ahuja A, Ying M. Sonography of neck lymph nodes. Part II: Abnormal lymph nodes. Clin Radiol 2003;58(5):359366. $\quad$ https://doi.org/10.1016/S00099260(02)00585-8

4. Gupta K, Chandra T, Venkatesan B, Tripathi P. Role of Color Doppler Ultrasound in Predicting Malignancy in Cervical Lymph Nodes. Int $j$ anat radiol surg 2016;5(4):15-20. Available from: $\quad$ http://www.ijars.net/articles/ PDF/2209/23816_CE[VSU]_F(GH) PF1(VsuGH)_PFA(GH)_PF2(VsuGH). pdf [Accessed 3rd August 2019].

5. Steinkamp HJ, Hosten N, Richter C, Schedel H, Felix R. Enlarged cervical lymph nodes at helical CT. Radiology 1994;191(3):795-798. $\quad$ https://doi. org/10.1148/radiology.191.3.8184067.

6. Ahuja A, Ying M. An Overview of Neck Node Sonography. Invest Radiol 2002;37:333-342. https://doi. org/10.1097/00004424-200206000$\underline{00005}$

7. Dangore SB, Degwekar SS, Bhowate RR. Evaluation of the efficacy of colour Dopplerultrasoundin diagnosis of cervical lymphadenopathy. Dentomaxillofac Radiol 2008;37:205-212. https://doi. org/10.1259/dmfr/57023901

8. Van den Brekel MW, Castelijns JA (2000)
Imaging of lymph nodes in the neck. Semin Roentgenol 2000;35(1):42-53. Available from: https://www.ncbi.nlm.nih.gov/ pubmed/10670052?dopt=Abstract\# [Accessed 9th August 2018]

9. Ariji $\mathrm{Y}$, Kimura $\mathrm{Y}$, Hayashi $\mathrm{N}$ et al. Power Doppler sonography of cervical lymph nodes in patients with head and neck cancer. AJNR Am J Neuroradiol 1998;19(2):303-7. Available from: https://pdfs.semanticscholar.org/9cac/ c360bb19b8ace9435f19719141654732a398.pdf [Accessed 5th August 2018].

10. Steinkamp HJ, Mäurer J, Comehl M, Felix R, Knöbber D, Hettwer H. Recurrent cervical lymphadenopathy: differential diagnosis with color-duplex sonography. Eur Arch Otorhinolaryngol 1994;251(7):404-409. $\quad$ https://doi. org/10.1007/BF00181966

11. Ahuja AT, Ying M, Ho SS, Metreweli C. Distribution of intranodal vessels in differentiating benign from metastatic neck nodes. Clin Radiol 2001;56(3):197$201 . \quad$ https://doi.org/10.1053/ $\underline{\operatorname{crad} .2000 .0574}$ 